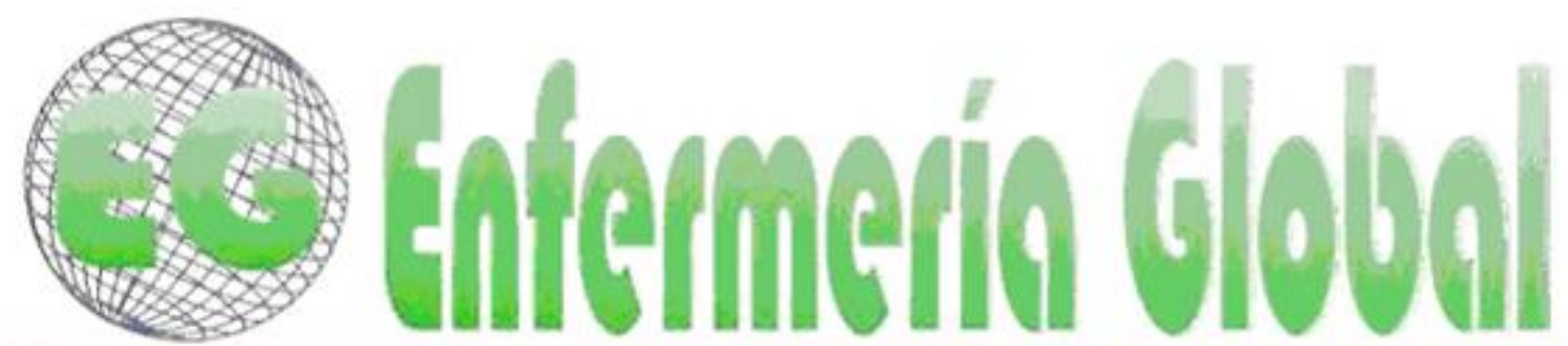

\title{
Trabajo Final de Grado y Plan de Acción Tutorial en el curriculum del Grado en Enfermería. Diseño, desarrollo y evaluación
}

Final Project Degree and Tutorial Action Plan in the curriculum of the Degree in Nursing. Design, development and evaluation

\section{${ }^{*}$ Roca Caparà, Núria *Morera Pomarede, María José *Roldán Merino, Juan ${ }^{*}$ Ramió Jofre, Anna}

*Profesor Titular, Grup d" investigació GIEES. EUI Sant Joan de Déu. Adscrita a la Universitat de Barcelona. España E-mail:: jroldan@santjoandedeu.edu.es

Palabras clave: Trabajo Fin de Grado; Grado en Enfermería; Plan de Acción tutorial; Tutoría universitaria

Keywords: Final Degree Project; Nursing Degree; Tutorial Action Plan; University Tutoring.

\section{RESUMEN}

La acción tutorial (PAT), en los estudios universitarios de Enfermería, se halla en el centro de la acción docente del profesorado. El tutor/a orienta en el proceso de aprendizaje y en la preparación del Trabajo Final de Grado (TFG). En este artículo presentamos los resultados de la investigación realizada con tutores y estudiantes, que tiene por objetivo evaluar el diseño y desarrollo del TFG, los resultados académicos y su vivencia y experiencia. Diseño mixto cuantitativo descriptivo y cualitativo de investigación acción participación. Muestra total de 124 estudiantes de 4o curso de Grado Enfermería y 37 tutores del TFG. Técnicas de recogida de datos: resultados académicos y evaluativos; 3 grupos focales con estudiantes y profesores; 3 entrevistas semiestructuradas abiertas a estudiantes. Los resultados constatan que las tipologías más elegidas fueron revisiones bibliográficas $(38,75 \%)$ y Planes de cuidados (24,2\%). El 63,9 \% de la temática escogida es Enfermería Maternal, Pediátrica y Clínica. Estudiantes y tutores viven con dificultad la elaboración del TFG y se requiere una mayor cultura tutorial. Es un proceso complejo de organización y coordinación que, sin embargo, permite un trabajo colaborativo de todo el equipo docente.

\section{ABSTRACT}

The Tutorial Action Plan (PAT) in the University School of Nursing, is central to the teaching action of professors. The tutor orients in the learning process and the preparation of the Final Degree Project (TFG). In this paper we present the results of research conducted with tutors and students, which aims to evaluate the design and development of the TGF, academic results and their experience and expertise. A descriptive qualitative and quantitative joint design of research and participation. A total sample of 124 students of 4th year in the nursing degree and 37 TGF tutor. Data collection techniques: 
Academic and evaluation results; 3 focus groups with students and professors; three semi-structured interviews open to students. Results indicate that the most commonly chosen typologies were literary reviews (38.75\%) and care plans (24.2\%). $63.9 \%$ of the chosen topics include Maternal, Pediatric and Clinical Nursing. Students and tutors struggle to develop the TGF and greater tutorial culture is required. It is a complex process of organization and coordination which, however, allows collaborative work of the entire faculty.

\section{INTRODUCCIÓN}

En el Campus Docent Sant Joan de Déu (Escola Universitària d'Infermeria, adscrita a la Universidad de Barcelona), la materia (asignatura) Trabajo Fin de Grado (TFG), de 6 créditos ECTS, se imparte en el $7^{0}$ semestre del Grado en Enfermería. Su inclusión en los planes de estudio se produce con la implantación del Grado en Enfermería (sustituye a la Diplomatura de 3 años), que estructura el curriculum atendiendo al marco normativo y metodológico del Plan Bolonia. Actualmente en España los estudios de Enfermería son grados universitarios de 4 años de duración.

La preparación y elaboración del TFG requieren un acompañamiento continuado por parte del equipo docente para favorecer que los estudiantes adquieran las competencias y resultados de aprendizaje propios de la titulación ${ }^{1}$.

En nuestra institución hemos diseñado el TFG de manera articulada al Plan de Acción Tutorial (PAT), que se desarrolla a lo largo de los cuatro cursos del Grado, como eje central y transversal del proceso de enseñanza-aprendizaje de los estudiantes. El tutor/a del PAT lo es también del TFG. La titularidad de la asignatura TFG corresponde a tres profesoras/es titulares doctores. Una de estas profesoras es la coordinadora del PAT. Los tutores del PAT-TFG son profesionales del ámbito docente, asistencial e investigadora.

Entendemos la tutoría como una actividad docente fundamental. El Plan de Acción Tutorial es el marco que regula la tutorización de los estudiantes y es una metodología estratégica de la acción formativa, cuya finalidad es favorecer la integración y participación de los estudiantes en la institución educativa, realizando un seguimiento personalizado del proceso de aprendizaje y orientándolo hacia su futuro profesional. En este sentido, el tutor y las tutorías del PAT orientan y tutelan la preparación y elaboración del TFG, a la vez que facilitan la adquisición de las competencias y de los resultados de aprendizaje propuestos en la materia.

El objetivo de este artículo es describir el proceso de implantación de la materia TFG, el análisis de su desarrollo y la evaluación de los resultados obtenidos en el primer año de docencia.

La tutoría es una estrategia didáctica básica de los procesos de enseñanzaaprendizaje y está considerada como un indicador de calidad de la práctica docente por nuestra institución. Entendemos que con ella se contribuye a la formación y desarrollo del estudiante universitario en todas sus dimensiones: académica, personal y profesional, siendo una herramienta esencial de la acción formativa ${ }^{2}$.

La acción tutorial, en los estudios universitarios, se halla en el centro de la acción docente y orientadora que debe realizar el profesorado. De acuerdo con los postulados de Rodríguez, la tutoría tiene por objetivo impulsar y facilitar el desarrollo integral de los estudiantes, permite personalizar la educación universitaria, asegura 
una atención continuada y próxima del estudiante, canaliza y dinamiza la integración y las relaciones del alumnado con la Institución ${ }^{3}$.

La comunicación y relación que se establece entre profesor y estudiante, durante las tutorías sirve para trabajar competencias y capacidades primordiales para la profesión enfermera: competencias relacionadas con las capacidades generales para el aprendizaje; habilidades y competencias relacionadas con el mundo profesional; habilidades comunicativas, relaciones interpersonales y desarrollo y compromiso personal $^{4}$.

El Espacio Europeo de Educación Superior (EEES) plantea, por una parte, que el estudiante sea protagonista activo y reflexivo de su aprendizaje, y por otra, que se reconozca su esfuerzo y dedicación en número de horas / créditos. Es un escenario que obliga al estudiante a implicarse y participar en su formación para consolidarla. En este sentido, la realización del TFG estimula la curiosidad por aprender y conocer, a la vez que potencia y reconoce la actividad autónoma del estudiante. El estudiante debe desarrollar por sí mismo las capacidades de búsqueda bibliográfica, lectura crítica, síntesis de contenidos, redacción científica del manuscrito y presentación oral del trabajo bajo la tutela del profesor/tutor. Es en esta asignatura donde mayoritariamente se pone de manifiesto el esfuerzo del estudiante y su autocapacidad de aprendizaje.

\section{DISEÑO DEL PLAN DOCENTE}

El TFG es una materia obligatoria de 6 créditos ECTS. Consiste en elaborar un trabajo individual que aglutine los conocimientos adquiridos en las diferentes materias que integran el Grado y los ámbitos de actuación propios de la disciplina enfermera.

Con la elaboración del TFG se pretende que los estudiantes sean capaces de:

- Identificar temas relevantes para la mejora de la profesión de Enfermería.

- Realizar búsqueda bibliográfica.

- Diseñar proyectos de investigación, programas de educación para la salud y planes de cuidados estandarizados.

- Desarrollar competencias y capacidades para la toma de decisiones, así como su desarrollo académico y profesional.

- Integrar la provisionalidad del conocimiento y la necesidad de la formación continuada.

- Identificar la importancia y necesidad de aprender de forma autónoma.

La tipología del trabajo incluye: revisión bibliográfica, programa de educación para la salud, plan de curas estandarizado o proyecto de investigación (cualitativa o cuantitativa). En diferentes asignaturas de primero, segundo y tercer curso, los estudiantes adquieren los conocimientos necesarios para realizar el TFG según estas tipologías.

Cada estudiante tiene asignado un tutor/a del Plan de Acción Tutorial, desde primer curso, que le orienta en todos los aspectos relacionados con el proceso de aprendizaje $y$, desde segundo curso, también en la preparación del TFG: metodología, contenidos, presentación y búsqueda bibliográfica. 
En cada curso académico, los estudiantes realizan un mínimo de 4 tutorías, 2 individuales y 2 grupales presenciales, además de tutorías virtuales según las necesidades individuales.

Para que estudiantes y tutores se basen en criterios uniformes hemos elaborado una Guía docente donde se detallan: reglamento y presentación del TFG; elección del tema y tipo de trabajo; criterios de presentación, formalización y defensa; criterios de evaluación; cronograma de elaboración y normas de presentación. También se especifica la estructura que debe tener cada tipología de trabajo. Además de la tutela individual del trabajo, impartimos seminarios teóricos grupales relacionados con su preparación y exposición.

Los coordinadores de la materia realizan tres reuniones grupales con el equipo de tutores de PAT/TFG a lo largo del curso académico.

La evaluación de la materia TFG es continuada e integral, ya que, tal como indican Coro y Martínez en Serrano ${ }^{1}$ tenemos en cuenta el proceso de preparación y el resultado final del trabajo. Para definir la cualificación final realizamos tres informes que aglutinan la evaluación de las competencias y resultados de aprendizaje asociados a la titulación:

- Informe de seguimiento del PAT / TFG que elabora el tutor/a del PAT y TFG. En este caso las rúbricas hacen referencia al interés, la actitud y el desarrollo del PAT y TFG. Tiene un valor del $30 \%$ de la nota final.

- Informe del trabajo escrito. Se evalúan la presentación, contenidos, metodología y bibliografía. Tiene un valor del $50 \%$ de la nota final.

- Informe de presentación oral. Evaluamos los conocimientos, la actitud y la presentación. Tiene un valor del $20 \%$ de la nota final.

Los informes del trabajo escrito y la presentación oral son realizados por los tribunales.

Entendemos que la evaluación conjunta de tutores y tribunales fomenta la participación, la toma de decisiones compartidas y el trabajo en equipo, ya que las guías de evaluación han pasado por un proceso de trabajo previo de debate y discusión grupal.

El equipo de tutores ha realizado formación en metodología docente, metodología de investigación y tutorización universitaria entre los años 2009 y 2013. Durante el curso 2012-13 hemos ampliado la formación con seminarios teóricos sobre las tipologías del TFG: revisión bibliográfica, diseño de un proyecto de investigación cualitativa / cuantitativa, programa de educación para la salud, plan de cures estandarizado.

\section{OBJETIVO GENERAL}

Evaluar el desarrollo del TFG y el Plan de Acción Tutorial como facilitador de la adquisición de las competencias y objetivos propuestos en el currículum. 


\section{OBJETIVOS ESPECÍFICOS}

1. Conocer las características sociodemográficas, tipologías de TFG y temáticas más frecuentes, así como los resultados académicos obtenidos por los estudiantes.

2. Conocer la opinión de los estudiantes en relación a la asignatura del TFG y el PAT.

3. Conocer la opinión de los tutores en relación a la formación continuada realizada.

4. Describir la vivencia de la elaboración del TFG de estudiantes y tutores.

\section{MÉTODOS}

Para analizar los datos se han utilizado dos metodologías diferenciadas.Por una parte se ha realizado un estudio cuantitativo de tipo descriptivo. Los participantes fueron los estudiantes que cursaban el 8o semestre del Grado en Enfermería del Campus Docent Sant Joan de Déu, Escuela Universitaria de Enfermería Adscrita a la Universidad de Barcelona y todos los tutores que tutorizaban estudiantes durante el curso académico 2012-13 (año en el que se implantó por primera vez la materia de TFG). Se recogieron variables relacionadas con el número de estudiantes matriculados en la asignatura y número de tutores del PAT que habían tutorizado el TFG, porcentaje de profesorado titular y colaborador, resultados académicos y opinión de los estudiantes en relación a la asignatura del TFG y del PAT. Además se analizó la formación continuada del equipo de tutores que participaron durante el proceso de tutorización.

Para conocer la opinión de los estudiantes se utilizaron dos cuestionarios diseñados específicamente para este estudio. El primer cuestionario recogía información en relación al grado de satisfacción con respecto a la organización de la materia del TFG y a los conocimientos, metodología, actitud y evaluación individual del profesorado. EI segundo cuestionario recogía información con respecto al Plan de Acción Tutorial (si las tutorías potencian la autonomía y responsabilidad del estudiante, si facilitan el aprendizaje práctico y teórico, si facilitan la adquisición de habilidades, hábitos y técnicas de estudio, utilidad de la carpeta de aprendizaje y la ayuda y acompañamiento del tutor durante el desarrollo del PAT). Por último, para conocer la opinión de los tutores que participaron en la formación continuada, se utilizó un cuestionario que recogía información en relación a la organización del curso, infraestructura, utilidad de la formación recibida, calidad del profesorado y calidad global de los contenidos.

Para cada una de las variables categóricas del estudio se calcularon frecuencias y porcentajes y para cada una de las variables cuantitativas la media y su desviación estándar. Se ha utilizado la prueba t de Student Fisher para estudiar la relación entre la nota media final obtenida por los estudiantes según el sexo y el turno del estudiante, y el tipo de tutor.

Se consideró estadísticamente significativa una $p$ bilateral inferior o igual a 0,05. El análisis de los datos se ha realizado mediante el programa PASW Statistics v.20.

Por otra parte, y con la finalidad de conocer la vivencia del TFG entre estudiantes y tutores, se ha realizado una investigación cualitativa de tipo educativa-evaluativa basada en un proceso de investigación - acción que tiene como finalidad mejorar la 
práctica docente. Se pretende obtener conocimiento aplicado que permita comprender el proceso educativo del TFG y lograr su mejora ${ }^{5}$. Se ha revisado, analizado y evaluado el desarrollo del Trabajo Final de Grado para obtener datos críticos con el fin de establecer un diagnóstico de sus carencias, debilidades y fortalezas, y de diseñar e implementar acciones de mejora.

Para la recogida de datos se realizaron dos grupos focales de tutores, un grupo focal de estudiantes de tercer y cuarto curso, y debate con los profesores que configuraron los tribunales para analizar todo el proceso e identificar propuestas de mejora. Las técnicas se desarrollaron una vez finalizó la presentación oral de los trabajos.

En los grupos focales participaron 12 tutores y 8 estudiantes. La elección de los estudiantes que participaron en el estudio se realizó con los siguientes criterios de inclusión: tener matriculadas asignaturas de tercer o cuarto curso, TFG en proceso de preparación o finalizado, estudiantes con diferentes tutores, estudiantes con buen desarrollo del PAT y estudiantes con dificultades en el desarrollo del PAT. Se buscó variabilidad de estudiantes para tener un debate más enriquecedor. En cuanto a los tutores, los criterios de inclusión fueron: formar parte del equipo de tutores desde el inicio del PAT. Se buscó la misma proporción de profesores titulares y colaboradores. Los temas de debate se plantearon en relación al diseño organizativo conjunto del PAT-TFG, la preparación del TFG, la formación metodológica de los tutores y el desarrollo de las tutorías.

Los datos se han analizado a través del análisis de contenido, codificando y categorizando la información más relevante en relación al objetivo planteado. Como estrategia analítica se utilizó la propia de la Teoría Fundamentada de Glaser y Strauss ${ }^{6}$ haciendo especial hincapié en el método comparativo constante. Se utilizó el recurso informático del programa Atlas/ti para el tratamiento de la información textual. $\mathrm{Se}$ establecieron estrategias específicas para asegurar los criterios de rigor. El proyecto fue aprobado por la Comisión de Ética Asistencial del centro, se pasó consentimiento informado a todos los participantes y se estableció un protocolo para asegurar la confidencialidad de los datos.

\section{RESULTADOS}

Con respecto a las características sociodemográficas de los estudiantes, el número total de estudiantes matriculados en el $8^{\circ}$ semestre de la asignatura del TFG fue de 124. El $65,3 \%$ de los mismos estaba matriculado en el turno de mañana y solamente el $10,5 \%$ eran chicos. El $21,8 \%$ de los estudiantes fueron tutorizados por profesores titulares y el resto por profesorado colaborador. Las revisiones bibliográficas $(38,75 \%)$ y los planes de cuidados estandarizados $(24,2 \%)$ fueron la tipología de trabajo más elegida por todos los estudiantes. Por lo que se refiere a los ámbitos temáticos, el $63,9 \%$ de todos los trabajos estaban relacionados con temas específicos de Enfermería de salud sexual y reproductiva, Enfermería Pediátrica y Enfermería Clínica. La nota media final (resultante de la nota del plan de acción tutorial, presentación oral y trabajo escrito) obtenida por los estudiantes fue de 7,6 (DE 1,1) siendo la nota mínima de un 5 y la nota máxima de un 9,7 (Tabla I). 
Tabla 1: Análisis descriptivo de las variables incluidas en el estudio

\begin{tabular}{|c|c|c|}
\hline Variables & n & $\%$ \\
\hline \multicolumn{3}{|l|}{ Sexo estudiante } \\
\hline Mujer & 111 & 89,5 \\
\hline Hombre & 13 & 10,5 \\
\hline \multicolumn{3}{|l|}{ Turno } \\
\hline Mañana & 81 & 65,3 \\
\hline Tarde & 43 & 34,7 \\
\hline \multicolumn{3}{|l|}{ TFG tutelados según tipología de profesor/a } \\
\hline Titular & 27 & 21,8 \\
\hline Colaborador & 97 & 78,2 \\
\hline \multicolumn{3}{|l|}{ Tipología del TFG } \\
\hline Revisión bibliográfica & 48 & 38,7 \\
\hline Proyecto de investigación & 23 & 18,5 \\
\hline Plan de cuidados estandarizado & 30 & 24,2 \\
\hline Programa de educación sanitaria & 23 & 18,5 \\
\hline \multicolumn{3}{|l|}{ Temática del TFG } \\
\hline Enfermería de salud sexual y reproductiva & 20 & 16,1 \\
\hline Enfermería Clínica & 37 & 29,8 \\
\hline Enfermería Gerontológica & 5 & 4,0 \\
\hline Enfermería de Salud Pública y Comunitaria & 12 & 9,7 \\
\hline Enfermería de Salud Laboral & 6 & 4,8 \\
\hline Enfermería del niño y adolescente & 29 & 23,4 \\
\hline Enfermería y Cuidados Paliativos & 6 & 4,8 \\
\hline Enfermería de Salud Mental & 8 & 6,5 \\
\hline Historia de la profesión & 1 & 0.8 \\
\hline \multicolumn{3}{|l|}{ Nota obtenida } \\
\hline PAT & \multicolumn{2}{|c|}{$8,2(\mathrm{DE} 1,6)$} \\
\hline Trabajo escrito & \multicolumn{2}{|c|}{$7,0(\mathrm{DE} 1,3)$} \\
\hline Presentación oral & \multicolumn{2}{|c|}{$8,1(\mathrm{DE} 1,2)$} \\
\hline Nota final & \multicolumn{2}{|c|}{$7,6(\mathrm{DE} 1,1)$} \\
\hline \multicolumn{3}{|l|}{ Resultados académicos de los estudiantes } \\
\hline Aprobados & 41 & 33,2 \\
\hline Notables & 64 & 51,6 \\
\hline Excelentes & 13 & 10,4 \\
\hline Matrícula de Honor & 6 & 4,8 \\
\hline
\end{tabular}

DE: desviación estándar

\section{Opinión de los estudiantes en relación al grado de satisfacción del TFG y del PAT}

Del total de 124 estudiantes matriculados, 120 estudiantes (96.7\%) respondieron al cuestionario de evaluación de la asignatura del TFG. En la tabla 2 se puede observar que la evaluación global de la materia obtiene una puntuación superior a 7 gracias a la evaluación individual del profesorado. La puntuación más baja se refiere a la organización de la asignatura.

Respecto a la opinión de los estudiantes en relación a PAT solamente 98 estudiantes contestaron el cuestionario, con una tasa de respuesta del $73.7 \%$. La Carpeta de Aprendizaje fue el ítem peor valorado por los estudiantes. Sin embargo, el acompañamiento del tutor y las tutorías fueron los aspectos mejor valorados por los estudiantes (tabla II). 
Tabla II: Opinión de los estudiantes en relación al Trabajo Final de Grado y Plan de Acción Tutorial

\begin{tabular}{|c|c|c|c|c|c|c|}
\hline \multirow[t]{2}{*}{ Ítems evaluados } & \multicolumn{2}{|c|}{ Total } & \multicolumn{2}{|c|}{$\begin{array}{l}\text { Grupo } \\
\text { mañana }\end{array}$} & \multicolumn{2}{|c|}{$\begin{array}{l}\text { Grupo } \\
\text { tarde }\end{array}$} \\
\hline & Media & $\mathrm{DE}$ & Media & $\mathrm{DE}$ & Media & $\mathrm{DE}$ \\
\hline \multicolumn{7}{|l|}{ Trabajo Final de Grado } \\
\hline Organización de la materia & 6.53 & 1.60 & 6.10 & 1.81 & 6.97 & 1.40 \\
\hline $\begin{array}{l}\text { Conocimientos, metodología, actitud del } \\
\text { profesorado }\end{array}$ & 6.88 & 1.80 & 6.55 & 1.92 & 7.21 & 1.69 \\
\hline Evaluación individual del profesorado & 8.15 & 1.40 & 8.05 & 1.54 & 8.26 & 1.26 \\
\hline Evaluación global de la materia & 7.19 & 1.66 & 6.90 & 1.76 & 7.48 & 1.57 \\
\hline \multicolumn{7}{|l|}{ Plan de Acción Tutorial } \\
\hline $\begin{array}{l}\text { Considero que la realización de las tutorías } \\
\text { contribuye a potenciar mi autonomía y } \\
\text { responsabilidad en el aprendizaje }\end{array}$ & 7.30 & 1.86 & 7.13 & 2.00 & 7.46 & 1.71 \\
\hline Me facilita el aprendizaje práctico y teórico & 6.90 & 2.14 & 6.75 & 2.28 & 7.05 & 2.00 \\
\hline $\begin{array}{l}\text { Me permite adquirir más conciencia de mi } \\
\text { aprendizaje y reflexionar sobre aquello que } \\
\text { estoy haciendo }\end{array}$ & 7.23 & 1.99 & 7.12 & 2.05 & 7.34 & 1.93 \\
\hline $\begin{array}{l}\text { Me facilita adquirir habilidades, hábitos y } \\
\text { técnicas de estudio más eficaces }\end{array}$ & 6.51 & 2.10 & 6.58 & 2.25 & 6.43 & 1.95 \\
\hline $\begin{array}{l}\text { Me ayuda en la adquisición de las } \\
\text { competencias y objetivos propuestos en las } \\
\text { asignaturas }\end{array}$ & 6.79 & 2.07 & 6.62 & 2.23 & 6.95 & 1.91 \\
\hline $\begin{array}{l}\text { La Carpeta de Aprendizaje es un buen } \\
\text { instrumento de formación y de construcción } \\
\text { de conocimiento }\end{array}$ & 6.44 & 2.33 & 5.97 & 2.30 & 6.91 & 2.35 \\
\hline $\begin{array}{l}\text { Considero que el tutor/a me ayuda en la mi } \\
\text { formación humana y profesional }\end{array}$ & 6.54 & 2.13 & 7.03 & 2.18 & 6.04 & 2.07 \\
\hline $\begin{array}{l}\text { El tutor/a me ha acompañado, asesorado y } \\
\text { orientado en mis estudios }\end{array}$ & 7.49 & 2.18 & 7.33 & 2.31 & 7.64 & 2.04 \\
\hline Total & 7.00 & 2.10 & 6.85 & 2.19 & 7.15 & 2.00 \\
\hline
\end{tabular}

DE: Desviación estándar

Se ha comparado la relación entre la nota media final obtenida por los estudiantes según el sexo y el turno de los mismos, y con el tipo de tutor, no encontrándose diferencias estadísticamente significativas (tabla III).

Tabla III: Relación entre la nota media final y el sexo del estudiante, el turno y tipo de tutor

\begin{tabular}{|c|c|c|c|}
\hline Variables & $\mathbf{n}$ & Media (DE) & $\mathbf{p}^{*}$ \\
\hline \multicolumn{4}{|l|}{ Sexo } \\
\hline Alumna & 111 & 7,6 (DE 1,1) & \multirow{2}{*}{0,486} \\
\hline Alumno & 13 & $7,4(\mathrm{DE} 1,2)$ & \\
\hline \multicolumn{4}{|l|}{ Turno } \\
\hline Mañana & 81 & $7,7(\mathrm{DE} 1,2)$ & \multirow[t]{2}{*}{0,140} \\
\hline Tarde & 43 & $7,4(\mathrm{DE} 1,0)$ & \\
\hline \multicolumn{4}{|c|}{ TFG tutelados según tipología de profesor/a } \\
\hline Colaborador & 97 & 7,5 (DE 1,5) & \multirow[t]{2}{*}{0,123} \\
\hline Titular & 27 & $7,9$ (DE 1,2$)$ & \\
\hline
\end{tabular}

DE: Desviación estándar; *t student Fisher; p: nivel de significación 


\section{Resultados de la formación continuada del equipo de tutores}

La formación se realizó en el primer semestre del curso académico y fue impartida por 4 profesores titulares del centro, especialistas en cada una de las tipologías de trabajo propuestas. Recibieron la formación 43 tutores/as (sobre 55 tutores de PAT) con un total de 12 horas. La formación fue evaluada positivamente por el equipo participante, destacando la utilidad de la formación recibida para tutorizar con más seguridad y preparación los TFG (tabla IV).

$\begin{aligned} & \text { Tabla IV: Valoración de los tutores participantes en la formación } \\
& \text { continuada }\end{aligned}$
\begin{tabular}{|l|c|c|}
\hline İtems evaluados & media & $\mathrm{DE}$ \\
\hline Adecuación del curso & 8.58 & 0.95 \\
\hline Infraestructura del curso & 8.71 & 0.89 \\
\hline Organización del curso & 9.00 & 1.08 \\
\hline Utilidad de la formación recibida & 9.08 & 1.11 \\
\hline Calidad global del profesorado & 9.13 & 0.97 \\
\hline Calidad global de los contenidos & 8.71 & 0.93 \\
\hline
\end{tabular}

DE: Desviación estándar

\section{Resultados de la segunda fase del estudio (cualitativa)}

La decisión de evaluar la implementación del TFG y del PAT después de su desarrollo completo responde a la necesidad de valorar la eficacia de su diseño en relación a la adquisición de las competencias propuestas; conocer las vivencias de sus actores principales, tutores y estudiantes; y detectar las áreas de mejora para aumentar su calidad. En general, podemos afirmar que el primer año de desarrollo de la asignatura TFG se caracterizó por la complejidad organizativa y las dificultades para unificar criterios de tutorización y evaluación entre tutores y tribunales.

A continuación se presentan los resultados más significativos de los grupos focales de estudiantes y tutores, así como de los debates realizados entre los miembros del claustro de tribunales.

A pesar de disponer de una Guía para la elaboración del trabajo escrito, de los seminarios impartidos a los estudiantes para la defensa oral ante el tribunal, de la formación continuada desarrollada con los tutores, de las reuniones del equipo docente y de las guías de evaluación, el sentimiento generalizado fue de incertidumbre y de dificultad,

"De hecho es lo más complicado. Deben leer, etc., etc... hasta que saben exactamente sobre qué lo harán (el TFG). Y esto es costoso. Hay gente que... o como muchos estudiantes que me encuentro yo, que les cuesta entender que primero tienen que hacer un trabajo previo para conseguir este tema, ¿vale? Entonces creen que dentro de unos días tendrán un tema fantástico, que será lo suficientemente pequeño o lo suficientemente grande, todo esto que decimos como para poderlo desarrollar. Si no lo han encontrado así, pues entonces voy apretando de alguna forma...: 'Digamos un tema, ¿no? De qué'. Y si no le haces caso es que no le estás orientando bien. Le estás diciendo: 'No, no, el trabajo es tuyo y yo te diré si vas mal o si vas bien', no?" (Tutor con estudiantes de $4^{\circ}$ curso) 
Los tutores participantes en los grupos focales destacaron su preocupación por orientar correctamente el TFG, tanto a nivel metodológico como de contenidos. También las dudas sobre el tema a escoger y la tipología del TFG generaron dificultades en el equipo de tutores en la orientación de los estudiantes. Los tutores se sintieron indirectamente evaluados por los tribunales, hecho que les generó, por una parte, mayor nivel de exigencia y respuesta ante los estudiantes, pero también mayor preocupación por los resultados finales de los trabajos. En este sentido, el proceso fue evaluado como de gran dificultad en su globalidad. También se valoró como un aspecto de dificultad añadida la falta de experiencia previa de los tutores para orientar de forma más eficaz a los estudiantes. Se estima que manteniendo el programa de formación continuada, el proceso mejorará con las posteriores promociones de estudiantes.

Los tutores valoraron positivamente, a nivel general, la dedicación y responsabilidad de la mayoría de estudiantes en la preparación del TFG aunque constataron una gran variabilidad en cuanto a la implicación en la preparación inicial del TFG, además de una gran dificultad para aglutinar y sintetizar los conocimientos teóricos adquiridos en cursos anteriores. En este sentido consideraron que las asignaturas teóricas que configuran la titulación son para muchos estudiantes conocimientos teóricos fragmentados que difícilmente interrelacionan y sintetizan en el TFG. Además, los tutores también destacaron el grado de exigencia de los estudiantes, ya que muchos esperan que sus tutores se impliquen totalmente y estén disponibles en todo momento, convirtiéndose (según la visión de los estudiantes) la orientación y tutorización en "elaboración" del trabajo.

Por su parte, muchos estudiantes reconocieron no haber concedido el valor ni la importancia que tiene en el Curriculum docente la realización del TFG. Admitieron no haber seguido los plazos recomendados para su preparación y, en algunos casos, dificultades para escoger tema, decidir tipología de trabajo y atender las recomendaciones del tutor/a, aunque hicieron una valoración positiva de la "libertad" para decidir la temática. Algunos estudiantes de cuarto curso señalaron como aspecto de dificultad, y que debe mejorarse, la necesidad de unificar mejor los criterios de tutorización y evaluación entre tutores del PAT/TFG y los profesores que configuran los tribunales. Refirieron que algunos tutores tuvieron dificultades para orientarlos a nivel metodológico y de contenidos, hecho que los estudiantes vivieron con preocupación y ansiedad. En este sentido, una de las observaciones de los estudiantes se refiere a la distinción entre profesores colaboradores y titulares. Opinaron que los profesores titulares tutorizaron mejor el TFG y los estudiantes obtuvieron mejores calificaciones.

A pesar de la valoración positiva que recibe la articulación del TFG en el PAT, muchos tutores y algunos estudiantes consideraron que el PAT queda condicionado desde segundo curso por el TFG y se desdibuja su objetivo prioritario. Por esta razón creen que se debe reforzar el PAT en si mismo, como una herramienta docente fundamental, en la que queda integrado el TFG. EI PAT es la esencia y el TFG es un elemento más en su desarrollo. Paralelamente entienden que el valor más importante que les aporta el PAT y el tutor/a es la tutorización del TFG. Subrayaron que el tutor/a les ayuda a planificar y gestionar mejor el tiempo. Resaltaron como aspecto más destacado que el tutor/a disponga de los conocimientos necesarios sobre metodología científica y praxis enfermera para una tutorización adecuada del TFG. En este sentido, algunos estudiantes se mostraron críticos con sus tutores/as cuando estos presentaron dificultades relacionadas con la organización y tutorización del TFG. Es 
significativo que muchos estudiantes hicieron autocrítica sobre su rendimiento general en la preparación del TFG:

"Yo sinceramente te digo que al principio lo dejaba un poco.... "iVa! ya lo haré, ya lo haré...." Y era, en ese sentido, era la tutora la que iba un poco detrás de mí ¿eh? Me decía: 'No llegamos. Ponte las pilas, tráeme algo...' Y claro, sí, yo trabajaba y ella me decía: 'Yo entiendo que tú trabajes pero los ratos libres ponte' ¿sabes? $Y$ en ese sentido muy bien, además de que al final ya me puse las pilas de tanto que me insistió, me insistía y puse ya de mi parte en plan deprisa y corriendo ¿sabes? Porque no llegábamos, pero en ese sentido yo sí que he sido un poquito relajado al principio y al final me fui poniendo las pilas, pero sí, me veo que podía haber dado más al principio y no ir tan ahogado la etapa final" (estudiante $4^{\circ}$ curso).

Tanto tutores de PAT y TFG como estudiantes consideraron fundamental transmitir la necesidad de investigar para mejorar la práctica asistencial. Creen que el TFG y las asignaturas con contenidos sobre investigación son instrumentos facilitadores para su consecución:

"La investigación que es muy importante en el mundo asistencial y ahora hay una falta brutal, o sea los que nos dedicamos a la asistencia, no hacemos investigación... Y entonces, inculcando al alumno que esto, esté donde esté, después o desarrolle su... su tarea, la que desarrolle... que hay una parte que es posible que desarrolle, o sea que la investigación es importante y que haga lo que haga... o sea, intentar hacerle ver la importancia, no?" (Tutora con estudiantes de 4ํㅡㄴ curso).

Desde el equipo coordinador se destacaron como aspectos positivos la disponibilidad, interés y responsabilidad de los tutores en la orientación y tutorización de los estudiantes durante los cuatro años de la titulación. Un elemento de dificultad detectado es el vínculo emocional y afectivo que se establece entre tutor/a y estudiante, hecho que en algún caso puede condicionar todo el proceso, principalmente la evaluación final que realiza el tutor:

[...] yo separaría lo que es el TFG de lo que es el PAT. Cuando tú tienes un alumno, lo recibes en primero y tú le preguntas, no sólo por la parte académica sino también por otros aspectos. A veces son aspectos personales de su vida, tú con este alumno tienes relación de ayuda o una relación que sí que es profesional pero bueno, hay elementos personales que se mezclan aquí. Entonces, cuando tú con este alumno lo llevas en el TFG, tienes una vinculación, no sólo para que lo juzguen tus compañeros o te evalúen a ti, sino que tú de este alumno conoces lo que le ha costado llegar allí. Tal vez no es un alumno de diez, pero ha sudado muchísimo..." (Tutora con estudiantes de $4^{\circ}$ curso).

Otro aspecto a destacar es el proceso de debate y reflexión que la implementación del TFG generó entre el equipo titular de la asignatura, la Comisión de Grado y el claustro de tribunales. El debate se centró en la práctica docente y la adquisición de competencias. También la organización y desarrollo de los tribunales, los claustros específicos sobre TFG y la realización de mejoras en el diseño durante el propio proceso fueron elementos valorados positivamente. Los aspectos identificados por tutores, estudiantes y tribunales, como susceptibles de mejora fueron: 
- Revisión de la Guía para la realización del TFG. Se detectó la necesidad de mejorar sus contenidos, especialmente la estructura que debe tener cada tipología de TFG.

- Profundizar la reflexión y unificar criterios en aspectos metodológicos, tanto en la tutorización como en la corrección de los trabajos.

- Potenciar la formación continuada de los tutores, ya que algunos refieren realizar el aprendizaje a medida que van tutorizando. En muchos casos se genera inseguridad y se requiere mucho esfuerzo y tiempo de dedicación.

- Implicar más y mejor a los estudiantes en los momentos iniciales de la preparación del TFG ya que estamos observando que en algunos casos lo retardan en exceso.

- Valorar la posibilidad y viabilidad de separar la tutorización del PAT y del TFG.

- Potenciar la formación de los estudiantes desde segundo curso en relación a las tipologías del TFG. Los estudiantes se sintieron desorientados porque carecen de experiencia en este tipo de trabajos. Les costó plantear el tema y su diseño porque lo inician cuando aun no han cursado todas las materias / asignaturas del currículum. Se constató que muestran más curiosidad por desconocimiento que por inquietud científica.

- Potenciar la cultura de la tutoría universitaria desde primer curso para asegurar un mejor resultado de todo el proceso formativo en la preparación del TFG ${ }^{?}$.

\section{DISCUSIÓN}

A pesar de las dificultades manifestadas por tutores y estudiantes en el desarrollo global del TFG, tanto el papel del tutor como la existencia del PAT en relación al TFG fueron valorados como una herramienta docente de alta calidad. Coincidiendo con Gidman, Humphreys, Andrews (2000) y Gidman (2001) todos los tutores valoraron la eficacia y beneficios de la tutorización para potenciar la reflexión de los estudiantes sobre su aprendizaje ${ }^{8,9}$. Nuestros tutores opinaron que la tutorización es un nuevo reto profesional que les obliga a formarse a nivel docente y metodológico para dar respuesta a las necesidades de los estudiantes.

La tutorización del TFG a través del PAT fue un aspecto muy valorado por los estudiantes de cuarto curso, una vez presentado el trabajo. La mayoría de ellos destacaron la buena relación con sus tutores y se manifestaron satisfechos con la experiencia ${ }^{10}$. Juzgaron positivamente tener un tutor/a que les oriente en la realización del PAT. Coincidiendo con las aportaciones de Consalter et $\mathrm{al}^{11}$, destacaron la experiencia de algunos tutores en el soporte y tutorización del TFG por su acompañamiento, orientación sistemática y experiencia.

Cuando la relación con el tutor/a se basó en la empatía y en el intercambio de opiniones, los estudiantes consideraron que las aportaciones del tutor fueron muy relevantes, siendo la comunicación y relación entre docente y discente fundamentales para el adecuado desarrollo del PAT ${ }^{4}$.

Como señala Zabalza ${ }^{2}$, un elemento que los estudiantes destacaron positivamente en el desarrollo del TFG fueron las tutorías grupales como proceso de formación. Un espacio de encuentro entre estudiantes de diferentes cursos que permite a los de tercero y cuarto compartir experiencias, inquietudes y dificultades. Se origina un clima favorable para que los estudiantes compartan sus vivencias y preocupaciones en relación al TFG. 
Los tutores del PAT-TFG participaron activamente en los seminarios de formación impartidos por el equipo titular y en las reuniones de reflexión e intercambio de opiniones. Esta participación aumentó el trabajo colaborativo del equipo docente y facilitó la investigación sobre la actividad desarrollada ${ }^{12}$.

Nuestra institución apuesta por la tutoría como una de las metodologías necesarias para garantizar la excelencia docente, a la vez que se crea una cultura universitaria basada en la tutoría como herramienta para la atención integral ${ }^{13,14}$.

No obstante, será necesario comparar esta evaluación con las posteriores promociones y valorar si los cambios operados en la organización de la misma permiten obtener un nivel de satisfacción más alto por parte de los estudiantes.

\section{CONCLUSIONES}

EI TFG es una síntesis de conocimientos, actitudes y habilidades integrados a lo largo de los cuatro cursos académicos, que el estudiante desarrolla para demostrar que ha adquirido las competencias transversales y específicas de la titulación. Su realización es fundamental como paso previo a la práctica profesional. Sin embargo, los estudiantes viven la asignatura, concretamente la realización del trabajo, como un reto y una gran dificultad porque es donde deben desarrollar y poner de manifiesto su capacidad de trabajo autónomo. No obstante, también refieren que a pesar de las dificultades, el proceso de elaboración y presentación les permite adquirir competencias profesionales que serán fundamentales para el ejercicio profesional, como la búsqueda de información, el análisis crítico, la toma de decisiones o la comunicación, entre otras. También toman consciencia de la importancia de investigar para mejorar el conocimiento y la praxis en Enfermería, además de ser capaces de diseñar Planes de cuidados estandarizados y Programas de educación para la salud fundamentales en la profesión.

La tutoría individualizada y el PAT, como componentes esenciales de la titulación, nos permiten realizar una orientación y seguimiento continuo del estudiante durante la preparación y elaboración del TFG. El diálogo entre tutor y estudiante permite conocer las características, preocupaciones, intereses y el proceso de desarrollo académico de este a lo largo de los cuatro cursos académicos. Docente y discente exploran con detenimiento las necesidades que se plantean, y a través de pactos, el tutor orienta y ayuda en la búsqueda de soluciones a problemas individuales, proponiendo las herramientas didácticas específicas para la realización del TFG.

A pesar de la complejidad que entraña la organización y coordinación articulada del TFG en el PAT, valoramos positivamente sus resultados por cuanto nos ha permitido personalizar la orientación de cada uno de nuestros estudiantes, realizar un trabajo cooperativo amplio (tutores y profesores titulares) y desarrollar acciones de mejora durante y al final del primer año de implantación de la asignatura. Sin embargo, debemos seguir trabajando en la evaluación y análisis continuado del diseño, desarrollo y resultados académicos de la materia TFG para que nuestros estudiantes sean capaces de lograr un aprendizaje óptimo, presentado trabajos de mejor calidad. Como docentes, debemos seguir investigando sobre nuestra práctica diaria para mejorar los planes docentes, las metodologías de aprendizaje, los instrumentos didácticos y la calidad de la Universidad en general. 


\section{REFERENCIAS BIBLIOGRÁFICAS}

1. Serrano Gallardo P. Trabajo fin de grado en ciencias de la salud . Madrid: DAE; 2012.

2. Zabalza Beraza MA. Competencias docentes del profesorado universitario: calidad y desarrollo profesional . Madrid:Narcea; 2006.

3. Rodríguez Espinar S, Álvarez González M. Manual de tutoría universitaria:recursos para la acción . Barcelona:Universitat de Barcelona, Institut de Ciències de l'Educació; 2004.

4. Miguel Díaz M de. Metodologías de enseñanza y aprendizaje para el desarrollo de competencias:orientaciones para el profesorado universitario ante el espacio europeo de educación superior . Madrid:Alianza; 2006.

5. Bisquerra Alzina R. Metodología de la investigación educativa . Madrid:La Muralla; 2004.

6. Glaser BG, Strauss AL. The Discovery of Grounded theory: strategies for qualitative research. Chicago: Aldine Pub. Co; 1967.

7. Casado R, Greca IM, Tricio V, Collado M, Lara AM. Impacto de un Plan de Acción

Tutorial universitario: resultados académicos, implicación y satisfacción. Rev Doc Universitaria. 2014;12(4):323-42.

8. Gidman J, Humphreys a, Andrews M. The role of the personal tutor in the academic context. Nurse Educ Today . $2000 ; 20(5): 401-7$.

9. Gidman J. The role of the personal tutor: a literature review. Nurse Educ Today . 2001;21(5):359-65.

10. Braine ME, Parnell J. Exploring student's perceptions and experience of personal tutors. Nurse Educ Today .2011;31(8):904-10.

11. Consalter L, Krahl M, Sain D, Barbosa C. A tutoria acadêmica no contexto histórico da educaçao. 2007;60(2):217-20.

12. Heyden M, Resck Z, Gradim C. A pesquisa na graduação em enfermagem: requisito para conclusão do curso. Rev Bras Enfermegagem. $2003 ; 56(4)$ :409-11. 13. González R. Modelo organizativo para la planificación y desarrollo de la Tutoría Universitaria en el marco del proceso de convergencia europea en Educación Superior. Rev Interuniv Form Profesorado. 2008 ;22(1):185-206.

14. Montes-Berges B, Morales A, Esteban F. La acción tutorial como innovación docente en la titulación de enfermería. Rev Electrónica Iniciación a la Investigación.2006;10:1-8.

Recibido: 19 de marzo 2015; Aceptado: 7 de mayo 2015

ISSN 1695-6141

(C) COPYRIGHT Servicio de Publicaciones - Universidad de Murcia 